Canadian

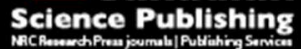

Canadian Journal of Forest Research Revue canadienne de recherche forestière

\title{
A NEURO-DYNAMIC PROGRAMMING APPROACH TO THE OPTIMAL STAND MANAGEMENT PROBLEM
}

\begin{tabular}{|r|l|}
\hline Journal: & Canadian Journal of Forest Research \\
\hline Manuscript ID & cjfr-2016-0302.R2 \\
\hline Manuscript Type: & Article \\
\hline Date Submitted by the Author: & $21-$ Feb-2017 \\
\hline Complete List of Authors: & $\begin{array}{l}\text { Comeau, Jules; Université de Moncton, Department of Administration } \\
\text { Gunn, Eldon; Dalhousie University }\end{array}$ \\
\hline Keyword: & Uncertainty, Forestry, NDP, Optimization, Policy \\
\hline \multicolumn{2}{|l}{} \\
\hline
\end{tabular}

SCHOLARONE $^{\text {In }}$
Manuscripts 
A NEURO-DYNAMIC PROGRAMMING APPROACH

TO THE OPTIMAL STAND MANAGEMENT PROBLEM

Halifax, NS, Canada, B3J 2X4

Halifax, NS, Canada, B3J 2X4

\footnotetext{
${ }^{1}$ Jules Comeau was a PhD student under the supervision of Eldon Gunn at the time of this study and is now a full time professor at Université de Moncton, Moncton, NB, Canada, E1A 3E9, email: jules.comeau@umoncton.ca. Eldon Gunn passed away on February 11, 2016 after a valiant battle with prostate cancer.
} 
10

11

12

13

14

15

16

17

18

19

20

21 22 and controls incorporated into our NDP model allows us to develop optimal policies with a level of

23 detail not typically seen in the forestry literature.

24 Keywords: uncertainty, forestry, NDP, policy, approximation

\section{ABSTRACT}

Some ideas of neuro-dynamic programming are illustrated by considering the problem of optimally managing a forest stand under uncertainty. Because reasonable growth models require state information such as height (or age), basal area, and stand diameter, as well as an indicator variable for treatments that have been performed on the stand, they can easily lead to very large state spaces that include continuous variables. Realistic stand management policies include silvicultural options such as precommercial and commercial thinning as well as post-harvest treatments. We are interested in problems that are stochastic in their basic growth dynamics, in market prices and in disturbances, ranging from insects to fire to hurricanes. Neuro-dynamic programming (NDP) algorithms are appropriate for problems with large dimensions that may lack a simple model of dynamics and stochastic processes. This paper looks at applying these ideas in the context of a multi species model. Results show that policies obtained using NDP are optimal within a 95\% confidence interval or better. The set of states detail not typically seen in the forestry literature. 


\section{INTRODUCTION}

Forestry is full of problems that involve the control of dynamic, stochastic systems with the intent to either optimize some objective function or to attempt to steer the system to some desired state. Even-age stand harvesting has been represented as a control problem (Näslund, 1969) and the classic (Faustmann, 1849) formula can be seen as a control policy developed from a deterministic view of the system. Dynamic programming (DP) has been used extensively to solve deterministic control problems in forestry and Yoshimoto et al. (2016) give an excellent overview of that literature. DP models in the forestry literature generally use some type of approximations to state and control spaces. For example, Lembersky and Johnson (1975) use 48 discrete stand indicants which were combinations of diameter at breast height and number of trees per hectare. In Haight and Holmes (1991), silvicultural actions are reduced to two discrete options: cut or no cut. Models use a selection of discrete values of forest stand descriptors and silvicultural interventions which are continuous in nature because realistic representations of those control problems are too complex to solve analytically.

Early applications of DP in forestry used a single continuous state variable such as age, volume, residual basal area or number of trees (Amidon \& Akin, 1968; Brodie \& Kao, 1979; Chen et al., 1980; Haight et al., 1985; Schreuder, 1971). However, to properly model growth and response to various silvicultural interventions, a single state variable is inadequate. Most growth models require a representation of something like stand average diameter, basal area, height as well as an indicator variable of stand nature (natural, plantation, pre-commercially thinned, commercially thinned, etc.); thus discrete and continuous variables. In representing multiple species stands, the state dimensions grow to include variables such as species percentage and crown closure.

Historically, most forestry studies limit the number of control options either because of limitations to the size of the model being used (Haight et al., 1985) or for simplicity (Lien et al., 
49

2007). Amidon and Akin (1968), Brodie et al. (1978), Brodie and Kao (1979), Haight et al. (1985), Arthaud and Klemperer (1988), Peltola and Knapp (2001) all applied methods that optimize the management of a stand over a set of decisions that keep the stand type as a natural or plantation through the entire optimization horizon without changing from one to the other, or do not differentiate between treatment condition. Treatment conditions reflect the history of the past decisions that have been made about the management of the stand. A list of practical silvicultural interventions usually includes some types of partial thinning, land preparation and final felling which include discrete and continuous variables. Combining them all in the same model would allow us to consider multiple treatment conditions simultaneously.

In forest management, decision-makers face highly uncertain outcomes because of the long time horizons (Hildebrandt \& Knoke, 2011) but risk is not the same as uncertainty. Risk has been defined as the expected loss due to a particular hazard thus uncertainty presents a risk if it results in an expected loss (von Gadow, 2001). Some risks such as loss of biodiversity are hard to evaluate financially but there is a very large literature that addresses financial risk and the sources of uncertainty that lead to expected losses. Readers should seek out review papers such as Hanewinkel et al. (2011), Hildebrandt and Knoke (2011) and Yousefpour et al. (2012). Textbooks such as Amacher et al. (2009) and Davis et al. (2005) are also excellent references.

Stochastic dynamic programming (SDP) has been used to investigate how control policies in a forestry context should be modified in the face of uncertain growth dynamics, market prices, interest rates, natural disasters and climate change (Ferreira et al., 2012; Haight \& Holmes, 1991; Hool, 1966; Lembersky \& Johnson, 1975; Rios et al., 2016; Steenberg et al., 2012; Yoshimoto, 2002)). In most studies, these uncertainties enter into forestry SDP models as transition probabilities between discrete states. For example, Ferreira et al. (2012) study the impact of wildfire occurrence by allowing a stand to transition from a non-burned to a burned / non-burned state according to 
73 transition probabilities $p$ and $(1-p)$ respectively, which depend on past wildfire history for that 74 stand.

75 Models with complex state spaces, dynamics and stochastic processes are becoming more common (Nicol \& Chadès, 2011; Rios et al., 2016; Steenberg et al., 2012). The amount and quality of data available to researchers and managers leads us to want to include more of that information into models. However, Bertsekas (2001) explains the limitations of SDP in handling large high dimensional continuous state spaces. When uncertainty leads to a large number of possible outcomes for each state-action pair, the number of calculations can quickly get out of hand for a problem with a large number of discrete values for a large number of state and control variables. In an effort to reduce the number of discrete states in a forestry context, Nicol \& Chadès (2011) use a heuristic sampling method that yields near-optimal policies. The control literature contains many examples of successfully using neuro-dynamic programming (NDP) to solve high dimensional problems in contexts other than forestry (Castelletti et al., 2007; Ilkova et al., 2012). The basic idea behind NDP is to evaluate the value function using a reduced number of discrete states and controls and approximate the value function and policy on the continuous portion of state and control spaces. A reduced number of discrete values for states and controls reduces the number of state-action pair outcomes for stochastic problems. Gunn (2005) describes the basics of NDP in a forestry context with the main ideas closely based on Bertsekas \& Tsitsiklis (1996) and Sutton \& Barto (1998). This paper expands on Gunn (2005) and presents more details about NDP implementation in a forestry context by using a case study of mix species even-aged stand management in Nova Scotia, Canada.

\section{PROBLEM DESCRIPTION}

The problem we want to solve is that of finding an optimal stationary policy $\mu$ of harvest and sylviculture for a two-species (red spruce and tolerant hardwood) even-aged stand growing in Nova Scotia, Canada. We have chosen to solve this problem by using a model that maintains an eight 
97 dimensional stand state $i$ : age, stocking (st), initial planting density (ipd), diameter for each species

$98\left(d^{\theta}\right)$, percentage of each species in terms of the proportion of total stand basal area $\left(p c t^{\theta}\right)$, crown 99 closure $(c c)$ which is directly proportional to spacing between trees and a discrete treatment 100 condition $(t t)$. $\theta$ refers to softwood (SW) or hardwood (HW). Any large open areas of a stand 101 would reduce stocking below 100\%. Crown closure is the percentage of ground, on the stocked 102 portion of the stand, covered by the vertical projection of tree crowns. Definitions of these variables 103 are those published in the national forestry database of silvicultural terms in Canada (1995). Each of 104 the first seven variables is continuous and can take on a wide range of values. A finite set $S^{\text {Eval }}$ of 105 1,127 discrete states $i$ is divided into five (5) subsets by treatment condition $S_{t t}^{\text {Eval }}, t t=1 \ldots .5$ because 106 growth and yield models differ depending on past treatment history. Table 1 shows the range of 107 values for state variables used in the model and which of the state variables are used for each treatment condition. In some instances, it will be necessary to use the following notation for states: $i_{N_{t t}}^{t t}$ where $N_{t t}$ is the number of discrete states for treatment $t t$. However, in order to simplify notation, where appropriate, we use $i$.

In this paper, uncertainty enters into the model as transition probabilities $p_{i j}(u)$ which represent uncertain market prices, uncertain impacts of natural disasters and, at harvest (clearcut), the stand is subject to an uncertain period of regeneration which can be eliminated through planting the stand. The financial risk associated with uncertain regeneration is the potential delay in growing stock being present on the stand thus delaying future harvests. Market prices are modeled as a stationary process and uncertain prices are assumed to follow a normal distribution with mean and variance chosen such that HW products are worth more than SW products and that there is enough difference between the highest and lowest prices for the policies to be different where price is a contributing 119 factor. There is no good long term market price data available for Nova Scotia, therefore we make 120 the assumption that the prices used in the model are reasonable. Haight (1990), Forboseh and 
121 Pickens (1996), and Lu and Gong (2003) are examples of studies that use stationary price models.

122 Because of their stationary nature, price is not included as a state variable in the model. The

123 financial risk associated with the uncertainty of market prices is the expected profit that could be

124 gained or lost by not harvesting in the current period and holding out for a better market price in a

125 future period. Natural disasters include hurricanes, forest fires and insect outbreaks. When a stand is 126 affected by a natural disaster, we model it as if it may or may not succumb to the disaster. If it does 127 not succumb, no damage is done and the stand remains unchanged. If it does succumb, we model this as if it will be completely wiped out and the value of the wood products on the stand would only be enough to pay for the cost of removing them, therefore the stand has no salvage value. Therefore, the financial risk associated with the uncertainty of natural disaster occurrence is the loss of potential future profit because the stand has succumbed before a planned harvest. Modelling hurricanes and fires is a matter of determining the probabilities of a stand ending up in any one of the regeneration states in any given period. Stands have a higher probability of succumbing to hurricanes as they get older and stems become more rigid and break more easily. In the case of fires, the opposite occurs as older trees have thicker bark that protects them from the heat. If an insect outbreak occurs, the stand will be affected and older trees are modeled to have a higher probability of succumbing as they offer better food and shelter for invading insects. Uncertain market prices, all three types of natural disasters and uncertain regeneration are assumed independent of each other.

Unplanted stands, once regenerated, grow as natural stands. Within certain diameter limits, they can be pre-commercially thinned (PCT) where a preference for one species will remove more of the

141 other. The forestry field handbook (1993) published by Nova Scotia Department of Natural 142 Resources (NSDNR) recommends spacing between $2.1 \mathrm{~m}$ and $3.0 \mathrm{~m}$ depending on the type and condition of the stand. Plantations can have either early competition control or not. Those without early competition control have a certain probability of reverting to a well-stocked natural stand. 
145 Plantations or PCT stands can be commercially thinned (CT) once they reach certain diameter limits.

146 In the case of $\mathrm{CT}$, there are a range of density reduction choices available with parameters including 147 thinning intensity (basal area removal of 20\%, 30\% and 40\%), species selection and removal method 148 (thinning from above, from below and across the diameter distribution). Basal area removal was 149 capped at $40 \%$ to protect the stand from windfall. Silvicultural options include those indicated above 150 as well as the decision to do nothing and let grow for one period. All of these management options 151 which include several continuous parameters are represented by 46 discreet options that form a finite 152 set $U(i)$ where feasible actions depend on $i$.

Growth models $f(i, u)$ are based on the Nova Scotia Softwood and Hardwood Growth and 154 Yield Models (GNY) (McGrath, 1993; O’Keefe \& McGrath, 2006). These models are based on extensive field analysis using a series of research permanent sample plots established and maintained by the N.S. Department of Natural Resources. The main parameter is site capability expressed as Site Index height at age 50. Models maintain height, diameter and basal area from period to period and equations were developed to translate model variables to GNY variables and vice-versa. For yield 159 calculations, merchantable and sawlog volumes are computed using Honer's equations (Nova Scotia Department of Natural Resources, 1996). GNY has essentially three growth models described below.

Natural unmanaged stands are grown using equations developed for the provincial revised 162 normal yield tables (Nova Scotia department of lands and forests, 1990). Age implies height (and 163 vice versa), height implies diameter and site density, which in turn implies basal area. Because the 164 model mixes two species together and because each species had different age-height relationships, 165 the model maintains age as a variable.

166 Natural stands that have been subject to pre-commercial and commercial thinning are grown 167 using a variable density model. The age-height relationship characteristic of the site is maintained. 168 The stand average diameters for each species are modeled as a function of site, spacing and free age. 
169 Free age is the mechanism that we use to deal with density modifications. This function is invertible 170 so that free age can be calculated as a function of site, spacing and diameter. Given a certain site, 171 spacing and diameter, we use the inverse function to compute the free age. If we choose to leave the 172 spacing as is, then growth amounts to increasing the free age by one period (five years) and 173 calculating the resulting diameter. A thinning alters both spacing and diameter. The nature of the 174 diameter change depends on the nature of the thinning (thinning from below, above or across the 175 diameter distribution). Given the new diameter and spacing, we can recompute free age and 176 continue to grow the stand by incrementing free age. Maximum density is a function of diameter and 177 diameter and basal area define both density and spacing. Trees are grown by advancing age (height 178 age) and free age by five years and recomputing diameter and height. Site density index (SDI) is 179 computed for this new diameter and, if current density exceeds SDI, current density is reduced to 180 SDI, spacing is recalculated and free age recomputed to correspond to the new diameter and spacing. 181 Plantations may be thinned and they are also grown using a variable density growth model but maximum density for plantations is computed using a different equation.

As a stand grows from its initial state and silvicultural prescriptions are applied, the stand may change from one treatment type to another. Applying silvicultural treatment $u$ to state $i$ results in state $j$ at the next time period, which are five year intervals because of the growth models used in Nova Scotia. For example, $t t=1$ represents natural stands which have been growing without intervention since the last final felling. Applying a pre-commercial thinning to this stand results in the stand transferring from $t t=1$ to $t t=3$, the latter of which is a treatment type for precommercially thinned natural stands. For growth purposes, there is no necessity to distinguish 190 between $t t=2$ and $t t=5$ or to distinguish between $t t=3$ and $t t=4$. However, the nature of revenue generated and silvicultural options are different in these circumstances. 
Rewards $g(i, u)$ are calculated based on volume extracted from a stand at period $k$ which is

193

194

195

196

197

198

199

200

201

202

203

204

205

206

207

208

209

210

211

212

213

dependent upon volume equations developed by NSDNR and on equations developed for this study. Market prices for merchantable and board volume for each species as well as silvicultural costs are factored in. The reader should see $g(i, u)$ as a profit function that calculates the total profit in any given time period of applying a silvicultural action $u$ to a forest stand $i$.

\section{METHODOLOGY}

There are many technicalities in properly detailing the nature of the problem to be solved which we will not present here but the notation used is closely based on Bertsekas and Tsitsiklis (1996) and interested readers should see Gunn (2005) for a succinct introduction to NDP. In light of our problem statement, it makes sense to think in terms of infinite horizon DP problems that yield stationary policies $\mu$ that map states $i$ into management decisions $u$. For any stationary policy $\mu$ and all states $i$, we have a discounted value function:

(1) $J^{\mu}(i)=\sum_{j} p_{i j}(\mu(i))\left[g(i, \mu(i))+\alpha J^{\mu}(j)\right]$

where $J^{\mu}(i)$ is the profit for state $i$ and policy $\mu, \mu(i)$ is the management decision for state $i$ according to policy $\mu$ and $\alpha$ is the discount factor. If we state $J^{*}(i)$ as an approximation to the optimal profit for state $i$, we are looking for a stationary policy $\mu$ that satisfies $J^{*}(i)=\max _{\mu} J^{\mu}(i)$ for all states $i$. The problem of finding an optimal stationary policy or finding $J^{*}(i)$ is usually approached by way of recursive approximation algorithms. One of three classes of algorithms is usually applied: value iteration, policy iteration and linear programming (Bertsekas \& Tsitsiklis, 1996). Policy iteration generates a sequence of stationary policies, each with improved profit over the preceding one. With a finite number of states and controls, policy iteration converges in a finite number of iterations but when the number of states and controls is large as it is the case in our problem, solving the linear system in the policy evaluation step of the policy iteration approach can 
214 be time consuming. Using approximate linear programming to solve a stochastic dynamic 215 programming forestry problem makes the linear programming problem difficult to handle given the 216 large number of variables and constraints (Bertsekas \& Tsitsiklis, 1996) therefore it will not be 217 discussed. Value iteration has been a standard method for solving DP and SDP problems in forestry 218 over five decades (Couture \& Reynaud, 2011; Hool, 1966). This paper presents the details of using 219 backward recursive value iteration in a NDP forestry problem for the first time.

Assuming that we know or can determine $p_{i j}(u)$ and $\alpha$, and that we have a closed form version of $f(i, u)$ and $g(i, u)$, we want to solve eq. (1) by way of a value iteration algorithm such as eq. (2) 222 where $k$ indexes iterations.

(2) $J_{k}(i)=\max _{u \in U(i)}\left\{\sum_{j} p_{i j}(u)\left[g(i, u)+\alpha J_{k-1}(j)\right]\right\}$

The recursive nature of eq. (2) requires approximations to be made as we may have a case where $j$ is not an element of $S^{\text {Eval }}$ and we do not have an exact value for $J_{k-1}(j)$. Figure 1 illustrates a value function $J$ plotted against a single state variable where the solid line represents the continuous nature of the value function, dots represent the values calculated at discreet states $i \in S^{E v a l}$ and X`s represent values approximated at discrete states $j$.

In the proposed approach, we replace $J_{k-1}(j)$ by $\tilde{J}_{k-1}(j, r)$ where $\mathrm{r}$ is the parameter vector of an approximation function. We rewrite eq. (2) as:

$$
J_{k}(i)=\max _{u \in U(i)}\left\{\sum_{j} p_{i j}(u)\left[g(i, u)+\alpha \tilde{J}_{k-1}(j, r)\right]\right\}
$$

The fitting approach that leads to the name neuro-dynamic programming is to let $r$ be the parameters of a neural network architecture. We generalize by letting $r$ be the parameters of any appropriate continuous function approximating architecture. For example, after calculating $J_{k}(i)$ for 
233 all $i \in S^{E v a l}$ at iteration $k=1$ of the algorithm, values of $J_{k}(i)$ are grouped by $t t$ and five separate 234 continuous functions $\tilde{J}_{k}^{t t}(i, r)$, one for each $t$, are fit on values of $J_{k}(i)$. At iteration $k=2$, these 235 continuous functions are used to calculate an approximation $\tilde{J}_{k-1}^{t t}(j, r)$ for the value of being in state $236 j$ which is of treatment condition $t t$. The sets of discrete states that describe each stand in each

The choice of an approximating architecture introduces a number of complications. The first is whether the approximating architecture $\tilde{J}_{k}(i, r)$ is capable of modeling the actual $J_{k}(i)$, the second is whether fitting of parameter vector $r$ has been successful in minimizing the fitting error, and the third is whether the $i_{1}^{t t}, i_{2}^{t t}, \ldots, i_{N_{t t}}^{t t}$ are numerous enough and chosen appropriately. Results in section 5.1 clearly demonstrate that Radial Basis Functions (RBF), presented below, answer affirmatively to each of these questions and can be an appropriate choice for NDP in a forestry context but care must be taken in choosing its parameters.

In this study, we use RBFs as function approximants which can be traced back to ideas put forward by Askey (1973) and further developed by Wendland (1995) and Buhmann (2000). RBFs have been successfully used for problems characterized by high dimensionality, in the hundreds in some cases, and can be incorporated into the NDP framework for value function approximations. RBFs are structured in such a way that the interpolant is forced to be equal to the function it is fitting at selected discrete points called centers and guarantees smooth transitions between points. Basis functions are radially symmetric about these centers which means their values depend only on the Euclidian distance from each center to each of the evaluation states, and on the shape of the basis function.

Four of the most mentioned forms of the RBF are the thin plate spline $\varphi=\delta^{2} \log \delta$, the multiquadric $\varphi=\left(\delta^{2}+\omega^{2}\right)^{1 / 2}$, the inverse multiquadric $\varphi=\left(\delta^{2}+\omega^{2}\right)^{-1 / 2}$, and the Gaussian 
$257 \varphi=e^{-\omega \delta^{2}}$ where $\omega$ is a shape parameter with its value set by the user, and $\delta$ is the scaled distance 258 between centers (Buhmann, 2000; Franke \& Schaback, 1998; Rippa, 1999). Each $\varphi$ yields a 259 different shape of the RBF function between centers. Distance between centers, calculated using a 260 Euclidian norm, plays a central role in RBFs. A sparse set of centers means a greater distance and a 261 higher probability that, between centers, the approximating function could behave in such a way that 262 it returns an approximation that is very different than the real value. The first two are probably the 263 best known and most often used versions of the RBF and this research reports results using the thin 264 plate spline which eliminates the need for finding a good value for $\omega$.

265 Before beginning the value iteration algorithm, we choose a subset of $M_{t t}$ states $i_{n}^{t t}, n=$ 266 $1, \ldots, M_{t t}$ from each subset $t t$ of $S^{E v a l}$ which we call centers. After having calculated $J(i)$ for all $267 i \in S^{E v a l}$ at iteration $k$ and having determined that the value iteration algorithm has not converged, 268 we force the interpolant $\tilde{J}^{t t}=J\left(i_{n}^{t t}\right)$ at the centers where $J\left(i_{n}^{t t}\right)$ are the values at those centers. $M_{t t}$ is 269 270 set as a function of the problem being solved and results will demonstrate the impact of choosing a different number of centers. The first step is to create, for every $t t$, a set of $M_{t t}$ equations, one for 271 each $i_{n}^{t t}$, using eq. (4).

(4) $J\left(i_{n}^{t t}\right)=\sum_{m=1}^{M_{t t}} r_{m}^{t t} \varphi\left(\left|i_{n}^{t t}-i_{m}^{t t}\right|_{p}\right)$ for $n=1 \ldots M_{t t}$

272 We know $\varphi$ and we use $p=2$ as the dimension of the Euclidian norm thus, for every $t t$, we have $273 M_{t t}$ unknowns, the $r_{m}^{t t}$, and $M_{t t}$ equations giving a linear system $A \times r=f$, where the elements of 274 the $M_{t t} \times M_{t t}$ square $A$ matrix are given by $\varphi\left(\left|i_{n}^{t t}-i_{m}^{t t}\right|_{2}\right)$ for $n=1 \ldots M_{t t}$, and the $M_{t t}$ elements of 275 vector $f$ are the values of $J\left(i_{n}^{t t}\right)$. In the cases where $\varphi$ is not defined for $i_{n}^{t t}=i_{m}^{t t}$, we set $\varphi=0$. If $A$ 276 is invertible, we have $r=f \times A^{-1}$ which yields the weights $r_{m}^{t t}$. Micchelli (1986) gives general 
277 conditions of $\varphi$ that ensure nonsingularity of $A$ and the RBF function chosen for implementation 278 meets those conditions.

279 Given the set of weights above, we can approximate the value of $J(j, r)$ at the next iteration of 280 the value iteration algorithm for any state $j$ according to the treatment type of state $j$ using eq. (5).

(5) $\tilde{J}^{t t}(j, r)=\sum_{m=1}^{M} r_{m}^{t t} \varphi\left(\left|j-i_{m}^{t t}\right|_{2}\right)$

Parameters $r_{m}^{t t}$ need to be recalculated at each iteration of the value iteration algorithm as they 282 depend on the updated values of $J(i)$.

283 We need to choose a method for terminating the value iteration algorithm. Bertsekas (2001) proposes a method, with accompanying mathematical proof, for terminating value iteration 285 algorithms that guarantees policy convergence as long as the stopping criteria $\varepsilon$ is sufficiently small.

Without discussing the details of the proof, the upper $\left(c_{k}^{U}\right)$ and lower $\left(c_{k}^{L}\right)$ bounds on the change of the value function for all evaluation states between iterations of the value iteration algorithm are 288 given by

$$
\begin{aligned}
& c_{k}^{L}=\frac{\alpha}{1-\alpha} \min _{i \in S^{E v a l}}\left[J_{k}(i)-J_{k-1}(i)\right] \\
& c_{k}^{U}=\frac{\alpha}{1-\alpha} \max _{i \in S^{E v a l}}\left[J_{k}(i)-J_{k-1}(i)\right]
\end{aligned}
$$

289 The value iteration algorithm is terminated when $c_{k}^{U}-c_{k}^{L} \leq \varepsilon$ and policy $\mu$ is an approximation to 290 the optimal policy. Results will show that policies converge before values with the stopping criteria $\varepsilon$ used in this study.

What follows is an outline of the NDP algorithm implemented in this study. Treatment indicator $t t$ is dropped to lighten notation but the reader is reminded that there is a function approximant for 294 each $t t$ and when approximating $J_{k-1}(j)$, the appropriate $\tilde{J}_{k-1}(j, r)$ must be used. 
295 Step 1: List discrete states $i \in S^{E v a l}$ and action set $U(i)$. Determine transition probabilities $p_{i j}(u)$, 296 GNY function $f(i, u)$, profit function $g(i, u)$ and discount factor $\alpha$. Set $k=1$ and go to step 2.

297 Step 2: Calculate $J_{k}(i)$ for all $i \in S^{E v a l}$ using eq. (2) and save optimal actions $u$ and $J_{k}(i)$ for future 298 retrieval. For $k=1, \tilde{J}_{k-1}(j, r)=0$ for all states $j$. For $k \geq 2$, use $\tilde{J}_{k-1}(j, r)$ to estimate $J_{k-1}(j)$ for 299 states $j$. Go to step 3.

300 Step 3: Stop if $c_{k}^{U}-c_{k}^{L} \leq \varepsilon$. Current policy $\mu$ is an approximation to the continuous optimal policy 301 and $J^{*}(i)$ are the values associated with $i \in S^{E v a l}$ and $\mu$. If $c_{k}^{U}-c_{k}^{L}>\varepsilon$, go to step 4 .

302 Step 4: Fit an RBF function $\tilde{J}_{k}^{t t}(i, r)$ for each $t t$ using values of $J_{k}(i)$ calculated at step 2 . Set $k=k$ $303+1$ and go to step 2 .

\section{POLICY VALIDATION}

There is no way of knowing the exact optimal policy as an analytical solution is not available thus it is important to remember that $\mu$ is a discrete approximation to the continuous optimal policy. While simulating $\mu$, it is probable that states will be visited for which a discrete action is not available thus forcing us to approximate $\mu$ for the continuous portion of the state space between discrete states. During a simulation replication, we use a nearest-neighbor scheme where we choose the action for the closest discrete state where distance is measured using the Euclidian norm.

Given the same uncertainties that were used in the NDP model, the main idea is to simulate $\mu$ for a sufficient number of replications to build a confidence interval (CI) for the expected NPV of current and future profits for a given initial state $i$. If the CI contains $J^{*}(i)$ from the NDP optimization, we conclude that discrete states in $S^{E v a l}$ were chosen appropriately, that value function approximations were consistent in the value iteration algorithm and that there is no reason to believe that $\mu$ is not a good approximation to the actual continuous optimal policy. A CI that does not include $J^{*}(i)$ indicates that there may be inconsistencies in the value iteration algorithm and $\mu$ should not be taken to be a good approximation to the optimal policy. 
What follows is a step by step description of how policy $\mu$ is simulated for state $i$ where $x_{t}$ is

320

321

322

323

324

325

326

327

328

329

330

331

332

333

334

335

336

337

used to represent state $i$. The simulation advances in 5 year increments and subscript $t$ represents the number of years since the beginning of the simulation replication.

Step 1: Choose state $x_{t}$ and simulation parameter values. Set $t=0$. Go to step 2 .

Step 2: Take action $u_{t}$ according to policy $\mu$ which results in state $x_{t+5}$ at time $t+5$ according to $\left(x_{t}, u_{t}\right)$. In the case of stochastic prices, generate a random number and determine prices to be used for selecting $u_{t}$ and for calculating $g_{t}\left(x_{t}, u_{t}\right)$. Calculate $N P V\left(g_{t}\left(x_{t}, u_{t}\right)\right)$. Go to step 3 .

Step 3: If a natural disaster occurs and the stand succumbs according to a generated random number, make state $x_{t+5}$ a regeneration state. Go to step 4 .

Step 4: If the stand is in a regeneration state, determine $x_{t+5}$ based a generated random number and regeneration probabilities. Go to step 5.

Step 5: Store $x_{t}, u_{t}, \operatorname{NPV}\left(g_{t}\left(x_{t}, u_{t}\right)\right)$ and $x_{t+5}$ for future retrieval. Go to step 6 .

Step 6: If $\operatorname{NPV}\left(g_{t}\left(x_{t}, u_{t}\right)\right)<\tau$ where $\tau$ is small enough to be financially inconsequential, stop the simulation. Otherwise, set $t=t+5$ and go to step 2 .

Steps 1 to 6 make up one simulation replication where $X^{m}=\sum_{t} N P V\left(g_{t}\left(x_{t}, u_{t}\right)\right)$ from that replication where $m$ is the replication number. When enough replications have been done for the same $i$ and $\mu$, an average and a standard deviation are calculated and are used to build a $1-\alpha$ confidence interval (CI) using the equations below:

$$
S^{2}(n)=\frac{\sum_{m=1}^{n}\left[X^{m}-\bar{X}\right]^{2}}{n-1} \quad \mathrm{CI}=\bar{X} \pm t_{n-1,1-\frac{\alpha}{2}} \sqrt{\frac{S^{2}(n)}{n}}
$$

where $\bar{X}$ is the average of $X^{m}$ for the $n$ replications and $t_{n-1,1-\frac{\alpha}{2}}$ is the value of the student- $t$ distribution. 
339 required to obtain an error of $\vartheta$ is given by Law and Kelton (2000):

$$
n^{*}(\vartheta)=\min \left\{a \geq n \text { such that } t_{a-1,1-\frac{\alpha}{2}} \sqrt{\frac{S^{2}(n)}{a}} \leq \vartheta\right\}
$$

343 We can determine $n^{*}(\vartheta)$ by iteratively increasing $a$ by 1 until a value of $a$ is obtained for which

$344 t_{a-1,1-\frac{\alpha}{2}} \sqrt{\frac{S^{2}(n)}{a}} \leq \vartheta$. In practice, the values of $X^{m}$ may not be exactly normally distributed therefore 345 Law and Kelton (2000) recommend the use of the $t$-distribution as it gives better coverage than a CI 346 constructed using the normal distribution.

347 Since each discrete state has its corresponding $\mu(i)$ and $J^{*}(i)$, any state can be chosen as the 348 starting point of the simulation and the corresponding $J^{*}(i)$ compared to the $(1-\alpha)$ CI above. Section 349 350

5.3 shows results of CIs constructed for a few discrete states and for different sets of parameters of the NDP model and discusses the absolute error $\vartheta$ for four simulations.

\section{RESULTS AND DISCUSSION}

All results in this section are for the base case scenario which uses the parameter values in table 2.

357 Distances between centers are calculated using the Euclidian norm with all values of variables scaled 358 to $0-1$. 
Inverting large ill-conditioned matrices can be difficult but scaling of the distances greatly

360

361

362

363

364 increases the condition number of the matrix which is an indication of the precision with which it can be inverted. Reducing the number of centers from a full basis, where all evaluation states are used as centers, to a reduced basis improves the condition number of the A matrix by many levels of magnitude. For example, for $t t=2$, we have 275 evaluation states. If we scale the distances between all these states and use all of them as centers in the RBF, the condition number of the matrix is $6.9496 \times 10^{7}$. This is in contrast with an A matrix with 37 centers evenly distributed over the range of values of the state variables which has a condition number of $5.8042 \times 10^{4}$, three orders of magnitude smaller. But the computational precision gained by having a much better conditioned A matrix comes at a great cost. Figures 2 and 3 show the difference between value function approximations using the RBF with 275 centers versus 37 centers.

In figure 2 , the full basis RBF makes less than $\$ 0.01$ in approximation error over the entire set $S_{2}^{E v a l}$. If the A matrix could be inverted without any loss of precision, those differences should all be null. By contrast, the approximation errors from the reduced basis RBF in figure 3 for those same 275 evaluation states range from $\$ 4,300$ to $-\$ 2,700$. Reducing the number of centers through which the RBF function is forced to pass, introduces errors in approximation at the evaluation states that were not included in the list of centers. Clearly, these approximation errors are not acceptable in a context of NDP where we are trying to iteratively build up values of being in any given state. For this reason, all results shown in this paper use the full basis thin plate spline RBF approximation architecture.

\subsection{Value iteration algorithm convergence}

All optimizations converged in a finite number of iterations and a few examples are presented below. Table 3 shows results for the base case scenario with the stopping criterion $\varepsilon=0.2$. There is a clear relationship between the discount rate and the number of iterations to convergence. Table 3 
383

384

385

386

387

388

389

390

391

392

393

394

395

396

397

398

399

400

401

402

403

404

405

406

shows the number of iterations to convergence versus the iteration number of the last policy change.

The iteration number shown in column three is the last iteration during which a policy change was recorded for any evaluation state. The fourth column of the same table shows the largest value function change for the last iteration where there is a policy change. Clearly, regardless of the discount rate, values have not converged when the policy stops changing thus supporting the claim that as long as $\varepsilon$ is sufficiently small, the value iteration algorithm will result in a stable policy that we show to be optimal through simulation in the next section.

\subsection{Monte Carlo policy simulation}

Table 4 gives the initial state for the four simulations discussed here. The first three simulations are done for softwood plantations because, given the parameters used in the NDP, all stands eventually become softwood plantations. It was important to use different discount rates as well as deterministic and stochastic prices. The fourth simulation starts with a young natural stand and allows us to simulate a larger portion of $\mu$.

Simulations 1 and 2 have three price levels so they require the use of random market prices. Confidence interval (CI) information for the four simulations is given in table 4 as is the number of replications in each simulation $\left(n^{*}(\vartheta)\right)$. CI`s are built using the average $\bar{X}$ and standard deviation for all replications $X^{m}$ for a given simulated $\mu$ and starting state $i$. Simulations 3 and 4 have one price level therefore the initial market state remains unchanged for the duration of each replication.

As recommended by Law and Kelton (2000), the confidence intervals for these simulations are constructed using a $95 \%$ confidence level and $\vartheta$ equivalent to $15 \%$ of the optimal value $J^{*}(i)$ from the NDP algorithm for the starting state $i$ being simulated. In all cases, confidence intervals contain $J^{*}(i)$ for starting state $i$ and policy $\mu$ but the intervals are quite wide because of the suggested large value of $\vartheta$. The $15 \%$ value suggested by Law and Kelton is very conservative for our purposes and leads to very wide confidence intervals that are not very useful. If $\vartheta$ was reduced until the $95 \% \mathrm{CI}$ 
407 was too narrow to include $J^{*}(i)$, we would have $\vartheta<5.2 \%$ for all simulations. We can say with $95 \%$

408 confidence and a relatively small absolute error, that $\mu$ is a good approximation to the continuous 409 optimal policy in those four cases.

\section{0}

411

412

413

414

\subsection{Policy discussion}

The policy discussion presented here is not meant to be a complete interpretation of the implications of using the policies calculated using NDP but rather some observations to reflect the level of detail obtained by using the methods described in this paper.

All states in the left side of table 5 are natural unmanaged stands $(t t=1)$ and they are discrete elements of $S^{E v a l}$, and all states in the right side of the table are either pre-commercially $(t t=2)$ or commercially thinned $(t t=5)$ stands. Each of the colour-coded numbers makes reference to an action to be applied to the stand. A pre-commercial thinning action takes the stand from $t t=1$ to $t t=2$ and a commercial thinning action applied to a natural unmanaged stand takes the stand from $t t=1$ to $t t=5$. The reader is reminded that the natural stands on the left have $100 \%$ crown closure on a $100 \%$ stocked stand therefore the stand is supporting as many trees as it possibly can for its age. These natural stands have an average SW content of $75 \%$. What follows is an example of the level of detail we can get from using NDP to develop individual stand management policies.

Action 1 is to do nothing and let the stand grow for one 5-year period. In all cases, taking action 1 simply means the stand will be 5 years older at the next decision time. For 5 year old natural stands, it is optimal to do nothing as the trees are too short to do a pre-commercial thinning and the diameters are too small to have any commercial value. Action 12 is a pre-commercial thinning that eliminates hardwood and spaces softwood to NS DNR recommended spacing and it is optimal to apply this action regardless of market price at ages 10 and 15. Taking action 12 with 10 and 15 year old natural stands, and letting them grow 5 years, results in transitions to the first two stands respectively (stands 1 and 2) in the right side of table 5. We notice that the new stands have had all 
431 their hardwood removed $\left(p c t^{S}=100 \%\right)$ and that the new crown closure percentage is very low. In

432 both cases, it is optimal to do nothing and let the stand grow (action 2) regardless of the observed

433 state of the market. As random disturbances occur and the state of the forest stand and market

434 evolve, the policies are used to continually make optimal decisions based on the state observed at 435 decision time.

436 By age 20, an unmanaged natural stand has self-thinned to a point that investing in a PCT to thin 437 out the stand is no longer the optimal action to take. Therefore, between the ages of 20 and 30 438 inclusively, it is optimal to do nothing and let the stand grow. At those ages, the average diameter of 439 the trees is still too small to have any commercial value.

440 All optimal CT actions remove $40 \%$ of the total basal area on the stand and, aside from a few exceptions, CT actions are clustered into two groups. The first group, actions 19, 28 and 37, all 442 remove the basal area by taking it as $25 \% \mathrm{SW}$ and $75 \% \mathrm{HW}$. The only difference is the manner in 443 which it is taken where 19 takes trees from the small diameters (from below), 28 takes the trees from 444 across the diameter distribution and 37 takes the trees from the largest diameters (from above). In the second group, actions 38 and 39, CT is done from above with 50\% basal area removal that is 75\% SW with the balance in HW. Doing a commercial thinning from above yields slightly higher 447 volumes for the same basal area but, more importantly, it creates a larger proportion of large logs which has a much higher market value, and higher market prices encourage the removal of larger 449 trees because there is a high probability that prices will come down at the next period. The majority 450 of the CT actions in the policy from the left hand side of table 5 lead to a state where the optimal 451 action is to do nothing for at least 5 years. Stands 3 to 13 from the right side of table 6 are a 452 sampling of the resulting forest stands after taking actions 19, 28, 37, 38 or 39 with the natural stands in the left side of table 5. Two characteristics are similar for all these stands: $p c t^{S}=100 \%$ for all 454 stands except stand 3 which still contains a small percentage of HW and $c c$ varies within a narrow 
455

456

457

458

459

460

461

462

463

464

465

466

467

468

469

470

471

472

473

474

475

476

477

478

range of $44 \%$ to $52 \%$. At such low $c c$, it makes no sense to remove any trees as the amount of

timber does not justify the removal. The policies in the right side of table 5 reflect this as it is optimal to do nothing for all stands up to 60 years of age (state 10). Starting at age 65, some regeneration harvests appear at very high prices with more appearing at age 75 (state 14). At this age, the stand diameter is high enough that it is optimal to do a regeneration harvest if the prices are simply above the mean. States 14 and 15 are shown to demonstrate that doing nothing when in state 14 yields state 15 , and that with the rise in $c c$ and diameter, there is a significant change in policy in just 5 years.

\section{CONCLUSION}

The main objective of this study as stated in the introduction is to demonstrate how to use a neuro-dynamic programming approach to the stand management problem when faced with high dimensional state and control spaces as well as multiple sources of uncertainty. For the same number of possible outcomes for each source of uncertainty, SDP typically requires a much higher number of discrete states because NDP can approximate the profit function between discrete states without sacrificing accuracy. In NDP, profit function approximations are built into the algorithm allowing for a much higher number of possible outcomes from all sources of uncertainty without needing to increase the number of discrete states. The set of state variables used in this study is not sufficient to fully model the management of real forest stands but it begins to approach the level of detail that forest managers might consider while developing policies. However, the detailed state/action spaces allow us to start considering the impact of uncertainty from sources such as the difference between planned harvest volumes and actual volumes, uncertain growth projections, climate change or uncertain quality of wood products at harvest.

Real world problems where many sources of uncertainty affect expected state/action outcomes and which would benefit from an additional level of detail in the optimal policies may be revisited using the ideas of NDP. Problems that could benefit from NDP typically are modeled using 
479 stochastic dynamic programming and have a state space that can be represented by a sparse set of 480 discrete states without loosing the level of detail necessary to capture policy changes. To see NDP 481 gain wider use in forestry, we must take a closer look at problems that would typically be solved 482 using SDP algorithms but would be modeled with a simplified representation of uncertainty in its 483 SDP form. The main reason for incorporating uncertainty in forestry models is to quantify risk in 484 order to make informed decisions. Methodologies such as NDP can capture the dynamics of real 485 decision making while simultaneously considering as many sources of uncertainty as possible so that 486 policies are reasonable representations of real life decisions and can be used to create useful policy 487 discussions.

$488 \quad$ Forestry will continue to pose important problems that involve the solution of large scale 489 stochastic dynamic programming problems. Neuro-dynamic programming is an approach that shows 490 considerable promise.

\section{Acknowledgements}

492 This work was partially supported through a Discovery Grant from the Natural Sciences and 493 Engineering Research Council of Canada. Dr. Jules Comeau was supported in part by a scholarship 494 from the Baxter and Alma Ricard Foundation.

\section{References}

495 Amacher, G. S., Ollikainen, M., \& Koskela, E. (2009). Economics of Forest Resources. Cambridge, 496 Massachussetts: MIT Press.

497 Amidon, E. L., \& Akin, G. S. (1968). Dynamic Programming to Determine Optimum Levels or $498 \quad$ Growing Stock. Forest Science, 14(3), 287-291. 
499 Arthaud, G. J., \& Klemperer, W. D. (1988). Optimizing high and low thinnings in loblolly pine with

500

501

502

503

504

505

506

507

508

509

510

511

512

513

514

515

516

517

518

519

520 dynamic programming. Canadian Journal of Forest Research, 18, 1118-1122. https://doi.org/10.1139/x88-172

Askey, R. (1973). Radial characteristic functions (Mathematics Research Center Report No. 1262). University of Wisconsin.

Bertsekas, D. P. (2001). Dynamic Programming and Optimal Control (2nd ed., Vol. 2). Belmont, Massachusetts: Athena Scientific.

Bertsekas, D. P., \& Tsitsiklis, J. N. (1996). Neuro-Dynamic Programming (2nd ed.). Belmont, Massachusetts: Athena Scientific.

Brodie, J. D., Adams, D. M., \& Kao, C. (1978). Analysis of Economic Impacts on Thinning and Rotation for Douglas-fir, Using Dynamic Programming. Forest Science, 24(4), 513-522.

Brodie, J. D., \& Kao, C. (1979). Optimizing Thinning in Douglas-fir with Three-Descriptor Dynamic Programming to Account for Accelerated Diameter Growth. Forest Science, 25(4), 665-672.

Buhmann, M. D. (2000). Radial Basis Functions. Acta Numerica, 9, 1-38.

Canadian Forest Service. (1995). Silvicultural Terms in Canada. Retrieved from http://nfdp.ccfm.org/terms/terms_e.php

Castelletti, A., de Rigo, D., Rizzoli, A. E., Soncini-Sessa, R., \& Weber, E. (2007). Neuro-dynamic programming for designing water reservoir network management policies. Control Engineering Practice, 15(8), 1031-1038. https://doi.org/10.1016/j.conengprac.2006.02.011

Chen, C. M., Rose, D. W., \& Leary, R. A. (1980). How to formulate and solve "optimal stand density over time" problems for even-aged stands using dynamic programming (No. General technical report NC-56) (p. 17). US Department of Agriculture Forest Service. 
521 Couture, S., \& Reynaud, A. (2011). Forest management under fire risk when forest carbon

522

523

524 525

$$
\text { sequestration has value. Ecological Economics, 70(11), 2002-2011. }
$$

$$
\text { https://doi.org/10.1016/j.ecolecon.2011.05.016 }
$$

Davis, L. S., Johnson, K. N., Bettinger, P., \& Howard, T. E. (2005). Forest Management To sustain Ecological, Economic, and Social Values (4th ed.). Long Grove, Il: Waveland Press Inc.

Department of Natural Resources of Nova Scotia. (1993). Forestry Field Handbook (p. 43).

$$
\text { Retrieved from http://novascotia.ca/natr/forestry/handbook/ }
$$

Faustmann, M. (1849). Calculation of the value which forest land and immature stands possess for forestry (Institute paper No. 42). Commonwealth Forest Institute, University of Oxford.

Ferreira, L., Constantino, M. F., Borges, J. G., \& Garcia-Gonzalo, J. (2012). A Stochastic Dynamic Programming Approach to Optimize Short-Rotation Coppice Systems Management Scheduling: An Application to Eucalypt Plantations under Wildfire Risk in Portugal. Forest Science, 58(4), 353-365. https://doi.org/10.5849/forsci.10-084

Forboseh, P. F., \& Pickens, J. B. (1996). A Reservation Value Approach to Harvest Scheduling with Stochastic Stumpage Prices. Forest Science, 42(4), 465-473.

Franke, C., \& Schaback, R. (1998). Solving partial differential equations by collocation using radial basis functions. Applied Mathematics and Computation, 93(1), 73-82. https://doi.org/10.1016/S0096-3003(97)10104-7

Gunn, E. A. (2005). A neuro-dynamic programming approach to the optimal stand management problem. In M. Bevers \& T. M. Barrett (Eds.), System analysis in forest resources: proceedings of the 2003 symposium (Vol. Gen. Tech. Rep. PNW-GTR-656, p. 366). Stevenson, Washington, US. Retrieved from http://www.fs.fed.us/pnw/publications/pnw_gtr656/ 
544 Haight, R. G. (1990). Feedback Thinning Policies for Uneven-Aged Stand Management with $545 \quad$ Stochastic Prices. Forest Science, 36(4), 1015-1031.

546 Haight, R. G., Brodie, J. D., \& Dahms, W. G. (1985). A Dynamic Programming Algorithm for 547 Optimization of Lodgepole Pine Management. Forest Science, 31(2), 321-330.

548

549

550

551

552

553

554

555

556

557

558

559

560

561

562

563

564

565

566

Haight, R. G., \& Holmes, T. P. (1991). Stochastic price models and optimal tree cutting: results for loblolly pine. Natural Resource Modeling, 5(4), 423-443.

Hanewinkel, M., Hummel, S., \& Albrecht, A. (2011). Assessing natural hazards in forestry for risk management: a review. European Journal of Forest Research, 130(3), 329-351. https://doi.org/http://dx.doi.org.proxy.cm.umoncton.ca/10.1007/s10342-010-0392-1

Hildebrandt, P., \& Knoke, T. (2011). Investment decisions under uncertainty-A methodological review on forest science studies. Forest Policy and Economics, 13(1), 1-15. https://doi.org/10.1016/j.forpol.2010.09.001

Hool, J. N. (1966). A Dynamic Programming - Markov Chain Approach to Forest Production Control. (E. L. Stone, Ed.) (Vol. 12). Washington, D.C.: Society of American Foresters.

Ilkova, T., Petrov, M., \& Roeva, O. (2012). Optimization of a Whey Bioprocess using NeuroDynamic Programming Strategy. Biotechnology \& Biotechnological Equipment, 26(5), 3249-3253. https://doi.org/10.5504/BBEQ.2012.0063

Law, A. M., \& Kelton, W. D. (2000). Simulation Modeling and Analysis (3rd ed.). McGraw Hill. Lembersky, M. R., \& Johnson, K. N. (1975). Optimal Policies for Managed Stands: An Infinite Horizon Markov Decision Process Approach. Forest Science, 21(2), 109-122.

Lien, G., Stordal, S., Hardaker, J. B., \& Asheim, L. J. (2007). Risk aversion and optimal forest replanting: A stochastic efficiency study. European Journal of Operational Research, 181, 1584-1592. https://doi.org/10.1016/j.ejor.2005.11.055 
567 Lu, F., \& Gong, P. (2003). Optimal stocking level and final harvest age with stochastic prices. 568 Journal of Forest Economics, 9(2), 119-136. https://doi.org/10.1078/1104-6899-00026 569 McGrath, T. (1993). Nova Scotia Softwood Growth and Yield Model - Version 1.0 User Manual 570 571 (Forest Research Report No. 43). Forest Research Section, Forestry Branch, N.S. Dept. of

\section{3}

574 575 Natural Resources. Retrieved from http://novascotia.ca/natr/library/forestry/reports/report43.pdf

Micchelli, C. A. (1986). Interpolation of Scattered Data: Distance Matrices and Conditionally positive definite Functions. Constructive Approximation, 2, 11-22. https://doi.org/10.1007/BF01893414

Näslund, B. (1969). Optimal Rotation and Thinning. Forest Science, 15(4), 446-451.

Nicol, S., \& Chadès, I. (2011). Beyond stochastic dynamic programming: a heuristic sampling method for optimizing conservation decisions in very large state spaces. Methods in Ecology and Evolution, 2(2), 221-228. https://doi.org/10.1111/j.2041-210X.2010.00069.x

Nova Scotia department of lands and forests. (1990). Revised normal yield tables for Nova Scotia softwoods (Research Report No. 22) (pp. 1-46). Nova Scotia Department of Lands and Forests. Retrieved from http://novascotia.ca/natr/library/forestry/reports/report22.pdf

Nova Scotia Department of Natural Resources. (1996). Honer’s Standard Volume Table Estimates Compared to Nova Scotia Stem Analyses. Retrieved from http://novascotia.ca/natr/library/forestry/reports/report61.pdf

O’Keefe, R. N., \& McGrath, T. P. (2006). Nova Scotia Hardwood Growth and Yield Model (No. FOR 2006-2 No. 78). Retrieved from http://novascotia.ca/natr/library/forestry/reports/REPORT78.PDF

Peltola, J., \& Knapp, K. C. (2001). Recursive Preferences in Forest Management. Forest Science, $47(4), 455-465$. 
591 Rios, I., Weintraub, A., \& Wets, R. J.-B. (2016). Building a stochastic programming model from

592

593

594

595

596

597

598

599

600

601

602

603

604

605

606

607

608

609

610

611

612

613

614 scratch: a harvesting management example. Quantitative Finance, 16(2), 189-199. https://doi.org/10.1080/14697688.2015.1114365

Rippa, S. (1999). An algorithm for selecting a good value for the parameter $\mathrm{c}$ in radial basis function interpolation. Advances in Computational Mathematics, 11, 193-210. https://doi.org/10.1023/A:1018975909870

Schreuder, G. (1971). The Simultaneous Determination of Optimal Thinning Schedule and Rotation for an Even-Aged Forest. Forest Science, 17, 333-339.

Steenberg, J. W. N., Duinker, P. N., \& Bush, P. G. (2012). Modelling the effects of climate change and timber harvest on the forests of central Nova Scotia, Canada. Annals of Forest Science, 70(1), 61-73. https://doi.org/10.1007/s13595-012-0235-y

Sutton, R. S., \& Barto, A. G. (1998). Reinforcement Learning (1st ed.). Cambridge, MA: MIT Press. Retrieved from https://mitpress.mit.edu/books/reinforcement-learning

von Gadow, K. (Ed.). (2001). Risk Analysis in Forest Management (1st ed., Vol. 1). Dordrecht / Boston / London: Kluwer Academic Publishers.

Wendland, H. (1995). Piecewise polynomial, positive definite and compactly supported radial functions of minimal degree. Advances in Computational Mathematics, 4(1), 389-396. https://doi.org/10.1007/BF02123482

Yoshimoto, A. (2002). Stochastic Control Modeling for Forest Stand Management under Uncertain Price Dynamics through Geometric Brownian Motion. Journal of Forest Research, 7, 81-90. https://doi.org/10.1007/BF02762512

Yoshimoto, A., Asante, P., \& Konoshima, M. (2016). Stand-Level Forest Management Planning Approaches. Current Forestry Reports, 2(3), 163-176. https://doi.org/10.1007/s40725-0160041-0 
615 Yousefpour, R., Jacobsen, J. B., Thorsen, B. J., Meilby, H., Hanewinkel, M., \& Oehler, K. (2012). A review of decision-making approaches to handle uncertainty and risk in adaptive forest management under climate change. Annals of Forest Science, 69(1), 1-15. https://doi.org/10.1007/s13595-011-0153-4

Table $1-$ Range of values for state variables where $S=$ softwood and $H=$ hardwood

\begin{tabular}{|c|c|c|c|c|c|c|c|c|c|c|}
\hline & \multicolumn{2}{|c|}{$\begin{array}{c}t t=1 \\
\text { Natural } \\
\text { unmanaged }\end{array}$} & \multicolumn{2}{|c|}{$\begin{array}{c}t t=2 \\
\text { PCT natural }\end{array}$} & \multicolumn{2}{|c|}{$\begin{array}{c}t t=3 \\
\text { Softwood } \\
\text { plantation }\end{array}$} & \multicolumn{2}{|c|}{$\begin{array}{c}t t=4 \\
\text { CT plantation }\end{array}$} & \multicolumn{2}{|c|}{$\begin{array}{c}t t=5 \\
\mathrm{CT} \text { natural }\end{array}$} \\
\hline & $\min$ & $\max$ & $\min$ & $\max$ & $\min$ & $\max$ & $\min$ & $\max$ & $\min$ & $\max$ \\
\hline Age (years) & 0 & 95 & 5 & 95 & 5 & 95 & 10 & 100 & 25 & 105 \\
\hline st \% & 75 & 100 & -- & -- & -- & -- & -- & -- & -- & -- \\
\hline $\begin{array}{c}i p d \\
\text { (trees/hectare) }\end{array}$ & -- & -- & -- & -- & 1000 & 4000 & -- & -- & -- & - - \\
\hline$d^{S}(\mathrm{~cm})$ & -- & -- & 5.3 & 30.8 & 0 & 28.1 & 3.9 & 33.4 & 8.5 & 37.2 \\
\hline$d^{H}(\mathrm{~cm})$ & -- & -- & 1.1 & 20.7 & -- & -- & -- & -- & 6.8 & 28.3 \\
\hline$p c t^{S}(\%)$ & -- & -- & 0 & 100 & -- & -- & -- & -- & 0 & 100 \\
\hline$c c(\%)$ & -- & -- & 5.7 & 100 & -- & -- & 40 & 100 & 40 & 100 \\
\hline \# discreet states & \multicolumn{2}{|c|}{38} & \multicolumn{2}{|c|}{275} & \multicolumn{2}{|c|}{76} & \multicolumn{2}{|c|}{90} & \multicolumn{2}{|c|}{648} \\
\hline
\end{tabular}

Table 2 - Basic parameters of the NDP model

\begin{tabular}{lr}
\hline Annual discount rate & $2 \%$ \\
\hline Site index SW (in meters at age 50) & $16.76 \mathrm{~m}$ \\
\hline Site index HW (in meters at age 50) & $17 \mathrm{~m}$ \\
\hline Minimum dominant stand height (hardwood) for doing a pre-commercial thinning & $6.1 \mathrm{~m}$ \\
\hline Minimum dominant stand height (softwood) for doing a pre-commercial thinning & $2 \mathrm{~m}$ \\
\hline Maximum average stand height for doing a pre-commercial thinning & $9 \mathrm{~m}$ \\
\hline Percentage of stand covered with softwood when a stand does naturally regenerate & $75 \%$ \\
\hline Natural stocking percentage of the forest & $75 \%$ \\
\hline Average selling price of softwood merchantable volume & $\$ 13.07 / \mathrm{m}^{3}$ \\
\hline Average selling price of softwood board volume & $\$ 30.76 / \mathrm{m}^{3}$ \\
\hline Average selling price of hardwood merchantable volume & $\$ 22.40 / \mathrm{m}^{3}$ \\
\hline Average selling price of hardwood board volume & $\$ 46.23 / \mathrm{m}^{3}$ \\
\hline Number of price level & 1 \\
\hline Cost of planting less than 2500 trees on one hectare & $\$ 1,350$ \\
\hline Cost of planting 2500 or more trees on one hectare & $\$ 1,500$ \\
\hline Cost of surveying one hectare of newly harvested land & $\$ 70$ \\
\hline Cost of doing fill planting on one hectare & $\$ 300$ \\
\hline Cost of doing pre-commercial thinning on one hectare & $\$ 750$ \\
\hline Cost of one hour of labour for doing commercial thinning or final felling & $\$ 40$ \\
\hline Flat cost of doing a commercial thinning on one hectare & $\$ 750$ \\
\hline
\end{tabular}




\begin{tabular}{lr}
\hline Approximate forested area in the west of the province of Nova Scotia (hectares) & $1,691,300$ \\
\hline Average number of fires per year in the area under study & 3.5 \\
\hline Return interval of major hurricanes (years) & 50 \\
\hline Average area of wind for a major hurricane (hectares) & 400,000 \\
\hline Return interval of major insect outbreaks (years) & 50 \\
\hline
\end{tabular}

Table 3 - Example results for the base case scenario with thin plate spline RBF approximation and stopping criterion $\varepsilon=0.2$

\begin{tabular}{cccc}
\hline$\alpha(\%)$ & $\begin{array}{c}\text { \# iterations for } \\
\text { convergence }\end{array}$ & $\begin{array}{c}\text { Iteration \# of last policy } \\
\text { change }\end{array}$ & $\begin{array}{c}\text { Largest value function change } \\
\text { at last policy change }\end{array}$ \\
\hline 1.0 & 138 & 105 & $\$ 110.55$ \\
\hline 2.0 & 101 & 42 & $\$ 287.11$ \\
\hline 4.0 & 39 & 23 & $\$ 14.11$ \\
\hline
\end{tabular}

Table 4 - Details of four Monte Carlo policy simulations including 95\% confidence interval and number of replications

\begin{tabular}{|c|c|c|c|c|c|c|c|c|c|c|}
\hline \multirow[t]{2}{*}{ Sim } & \multirow[t]{2}{*}{$\alpha$} & \multirow{2}{*}{$\begin{array}{c}\text { \# of price } \\
\text { breaks }\end{array}$} & \multirow{2}{*}{$\begin{array}{l}\text { Replication } \\
\text { length }\end{array}$} & \multicolumn{3}{|c|}{ Starting state } & \multirow[t]{2}{*}{ Lower CI } & \multirow[t]{2}{*}{ Upper CI } & \multirow{2}{*}{$\begin{array}{l}\text { DP value } \\
\text { function } \mathrm{J}^{*}\end{array}$} & \multirow[t]{2}{*}{$n^{*}(\vartheta)$} \\
\hline & & & & TRT & Age & $\begin{array}{l}\text { Initial } \\
\text { density }\end{array}$ & & & & \\
\hline 1 & 0.04 & 3 & 295 years & 3 & 25 & 2500 & $\$ 2,838.79$ & $\$ 3,862.56$ & $\$ 3,403.40$ & 29 \\
\hline 2 & 0.04 & 3 & 295 years & 3 & 35 & 3250 & $\$ 3,263.89$ & $\$ 10,376.53$ & $\$ 6,945.36$ & 2 \\
\hline 3 & 0.03 & 1 & 295 years & 3 & 30 & 2500 & $\$ 4,352.78$ & $\$ 6,254.93$ & $\$ 4,890.85$ & 4 \\
\hline 4 & 0.02 & 1 & 695 years & 1 & 15 & $\mathrm{n} / \mathrm{a}$ & $\$ 9,606.29$ & $\$ 12,998.07$ & $\$ 11,399.81$ & 19 \\
\hline
\end{tabular}


Table 5 - Partial policies for the base case scenario with 6 price levels

\begin{tabular}{|c|c|c|c|c|c|c|c|c|c|c|c|c|c|c|c|c|c|c|c|}
\hline \multirow[b]{2}{*}{ 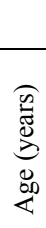 } & \multicolumn{7}{|c|}{ Natural unmanaged stands } & \multicolumn{12}{|c|}{ Commercially or pre-commercially thinned natural stands } \\
\hline & 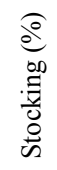 & 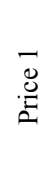 & 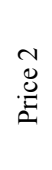 & $\stackrel{0}{0}$ & $\begin{array}{l}\stackrel{+}{\Xi} \\
\stackrel{\Xi}{\Xi}\end{array}$ & $\begin{array}{l}n \\
\ddot{D} \\
\stackrel{0}{D}\end{array}$ & 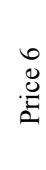 & $\begin{array}{l}\dot{\bar{D}} \\
\text { है } \\
\bar{\Xi} \\
\bar{\Xi} \\
\text { 芯 }\end{array}$ & 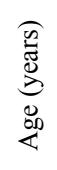 & $\underbrace{\widehat{E}}_{\tilde{z}}$ & 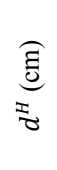 & 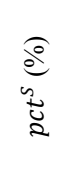 & $\underbrace{0}_{\tilde{u}}$ & 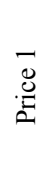 & 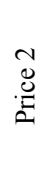 & $\begin{array}{l}n \\
\mathscr{0} \\
\tilde{E}\end{array}$ & 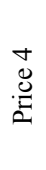 & $\begin{array}{l}n \\
\ddot{0} \\
\ddot{E}\end{array}$ & $\begin{array}{l}0 \\
.0 \\
:\end{array}$ \\
\hline 5 & 100 & 1 & 1 & 1 & 1 & 1 & 1 & 1 & 15 & 5.2 & 0 & 100 & 8.4 & 2 & 2 & 2 & 2 & 2 & 2 \\
\hline 10 & 100 & 12 & 12 & 12 & 12 & 12 & 12 & 2 & 20 & 6.9 & 0 & 100 & 14.1 & 2 & 2 & 2 & 2 & 2 & 2 \\
\hline 15 & 100 & 12 & 12 & 12 & 12 & 12 & 12 & 3 & 40 & 8.7 & 7.0 & 92 & 46.5 & 2 & 2 & 2 & 2 & 2 & 2 \\
\hline 20 & 100 & 1 & 1 & 1 & 1 & 1 & 1 & 4 & 45 & 10.6 & 0 & 100 & 44.5 & 2 & 2 & 2 & 2 & 2 & 2 \\
\hline 25 & 100 & 1 & 1 & 1 & 1 & 1 & 1 & 5 & 50 & 12.1 & 0 & 100 & 44.7 & 2 & 2 & 2 & 2 & 2 & 2 \\
\hline 30 & 100 & 1 & 1 & 1 & 1 & 1 & 1 & 6 & 55 & 13.5 & 0 & 100 & 44.9 & 2 & 2 & 2 & 2 & 2 & 2 \\
\hline 35 & 100 & 1 & 1 & 39 & 39 & 39 & 39 & 7 & 55 & 15.0 & 0 & 100 & 51.1 & 2 & 2 & 2 & 2 & 2 & 2 \\
\hline 40 & 100 & 1 & 38 & 38 & 38 & 38 & 38 & 8 & 55 & 14.0 & 0 & 100 & 51.6 & 2 & 2 & 2 & 2 & 2 & 2 \\
\hline 45 & 100 & 38 & 38 & 38 & 38 & 38 & 38 & 9 & 60 & 15.0 & 0 & 100 & 45.0 & 2 & 2 & 2 & 2 & 2 & 2 \\
\hline 50 & 100 & 19 & 19 & 37 & 38 & 38 & 38 & 10 & 60 & 15.4 & 0 & 100 & 51.8 & 2 & 2 & 2 & 2 & 2 & 2 \\
\hline 55 & 100 & 37 & 37 & 37 & 37 & 38 & 7 & 11 & 65 & 16.8 & 0 & 100 & 51.9 & 2 & 2 & 2 & 2 & 2 & 7 \\
\hline 60 & 100 & 28 & 37 & 37 & 37 & 7 & 7 & 12 & 70 & 18.2 & 0 & 100 & 52.1 & 2 & 2 & 2 & 2 & 2 & 7 \\
\hline 65 & 100 & 37 & 37 & 37 & 7 & 7 & 7 & 13 & 75 & 19.4 & 0 & 100 & 52.2 & 2 & 2 & 2 & 7 & 7 & 7 \\
\hline 70 & 100 & 1 & 37 & 7 & 7 & 7 & 7 & & & & & & & & & & & & \\
\hline 75 & 100 & 1 & 1 & 7 & 7 & 7 & 7 & 14 & 65 & 17.4 & 0 & 100 & 51.7 & 2 & 2 & 2 & 2 & 2 & 7 \\
\hline 80 & 100 & 1 & 1 & 7 & 7 & 7 & 7 & 15 & 70 & 22.0 & 0 & 100 & 59 & 2 & 2 & 2 & 7 & 7 & 7 \\
\hline 85 & 100 & 1 & 1 & 7 & 7 & 7 & 7 & & 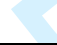 & 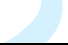 & & & & & & & & & \\
\hline 90 & 100 & 1 & 1 & 7 & 7 & 7 & 7 & 16 & 100 & 27.2 & 0 & 100 & 52.0 & 2 & 2 & 7 & 7 & 7 & 7 \\
\hline
\end{tabular}

\begin{tabular}{lllllllll}
95 & 100 & 19 & 19 & 7 & 7 & 7 & 7 \\
\hline
\end{tabular}

1 - Let grow

2 - Let grow

7 - ReHar, plt 2500 tr/ha
12 - PCT, remove HW

19 - CT, rmv $40 \%$ BA, splt $25 \%$ (blw)

28 - CT, rmv $40 \%$ BA, splt $25 \%$ (cros)
37 - CT, rmv $40 \%$ BA, splt $25 \%$ (abv)

38 - CT, rmv $40 \%$ BA, splt $50 \%$ (abv) 39 - CT, rmv $40 \%$ BA, splt $75 \%$ (abv) 
Figure 1 - Representation of a value function. The solid line represents the continuous nature of the value function, the dots represent the values calculated at a discreet states $i \in S^{\text {Eval }}$ and X's represent values approximated at discrete states $j$.

Figure 2 - Difference between approximate value and actual value, plotted against age, for the 275 discrete states for TRT $=2$ with those 275 states being used as centers in the RBF with distances scaled to $0-1$

Figure 3 - Difference between approximate value and actual value, plotted against age, for the 275 discrete states for TRT $=2$ with 37 states being used as centers in the RBF with distances scaled to 01 
Figure 1

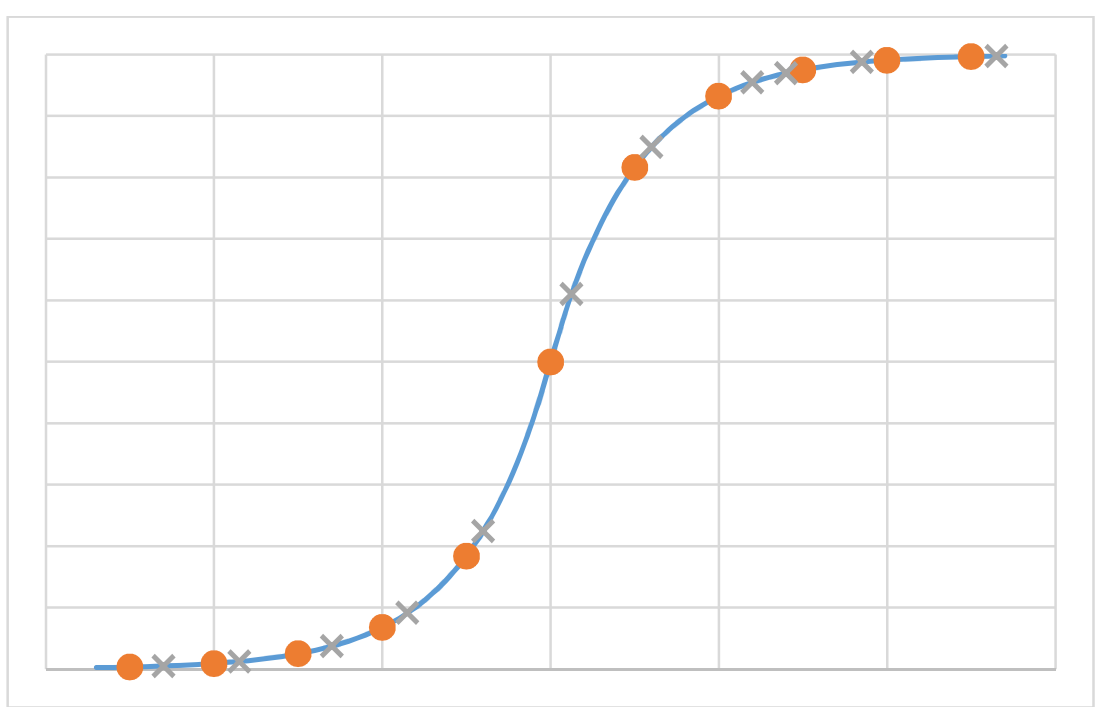

Figure 2

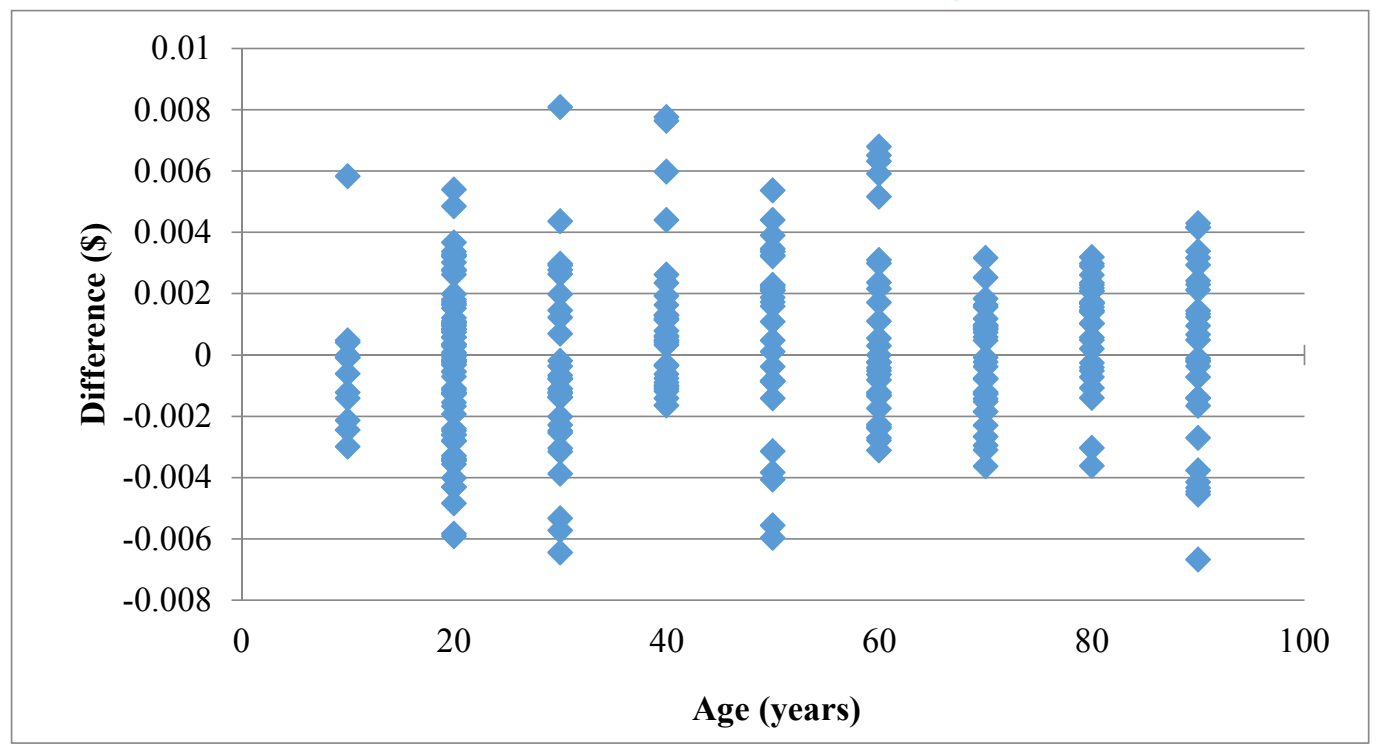

https://mc06.manuscriptcentral.com/cjfr-pubs 


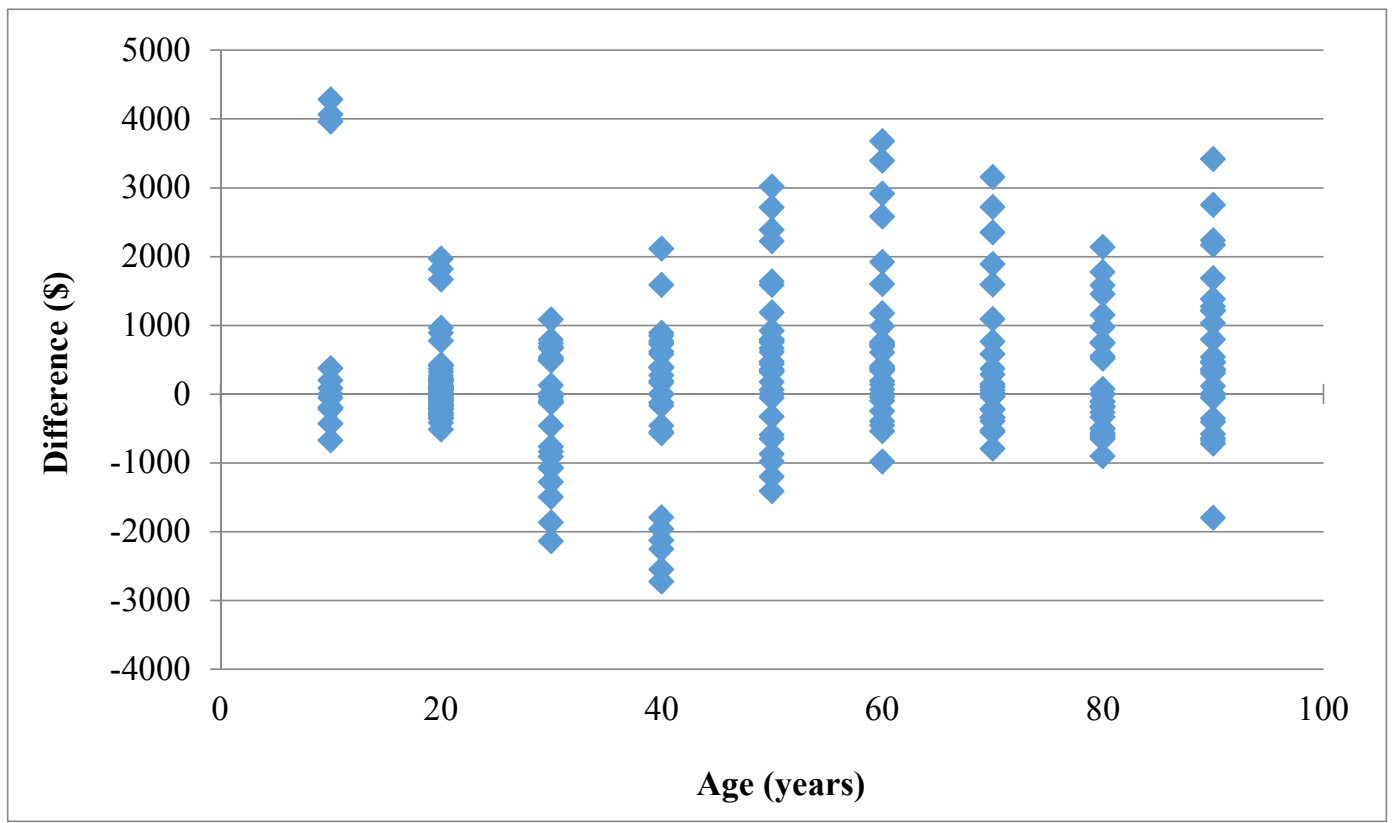

Figure 3 